\title{
Age should not be a limiting factor in laparoscopic surgery: a prospective multicenter cohort study on quality of life after laparoscopic hysterectomy
}

This article was published in the following Dove Press journal:

Clinical Interventions in Aging

\author{
Justine M Briët' \\ Marian JE Mourits' \\ Barbara L van Leeuwen ${ }^{2}$ \\ Edwin $\mathrm{R}$ van den Heuvel $^{3}$ \\ Monique JA Kenkhuis' \\ Henriette JG Arts' \\ Geertruida $\mathrm{H}$ de Bock ${ }^{3}$ \\ 'Department of Gynecologic \\ Oncology, University Medical Center \\ Groningen, University of Groningen, \\ Groningen, the Netherlands; \\ ${ }^{2}$ Department of Surgical Oncology, \\ University Medical Center Groningen, \\ University of Groningen, Groningen, \\ the Netherlands; ${ }^{3}$ Department of \\ Epidemiology, University Medical \\ Center Groningen, University \\ of Groningen, Groningen, the \\ Netherlands
}

Correspondence: Justine M Briët Department of Gynecology, University of Groningen, University Medical Center Groningen, Hanzeplein I, 9700RB Tel +3I 503613152

Email j.briet@zgt.nl Groningen, the Netherlands

Purpose: A prospective, multicenter cohort study was performed on the implementation of laparoscopic hysterectomy (LH) in the Netherlands. The aim of this study was to evaluate the impact of LH on quality of life (QOL) with respect to age up to 6 months postoperatively.

Patients and methods: Women with an indication for LH, either for benign conditions or early-stage low-risk endometrial cancer were included. QOL was measured before and 6 weeks and 6 months after surgery, using the 36-item Short Form Health Survey. Mean QOL values were compared to an unselected, female, Dutch reference population. A longitudinal linear mixed model was applied to assess changes in QOL over time after LH and to determine if in patients $\geq 65$ years of age QOL scores were different.

Results: Data on 116 patients were available for analysis. The median age was 54 years at time of surgery (range 34-83) with an interquartile range of 43-65 years. Six months after LH, all QOL values were higher than before surgery and were equal to or higher than those of the reference population. Older women tend to score higher on QOL preoperatively, and these scores remain high postoperatively.

Conclusion: After LH, QOL improves. Older women report higher QOL values preoperatively and QOL is still high 6 months after the operation. Age does not confer a negative impact on QOL following LH and should not be the reason to refrain from laparoscopic surgery.

Keywords: quality of life, laparoscopic surgery, hysterectomy, elderly

\section{Introduction}

Surgery is becoming more and more minimally invasive. In standard surgical procedures such as appendectomy or cholecystectomy, most patients are operated on laparoscopically. ${ }^{1,2}$ Minimally invasive procedures tend to have little negative impact on quality of life (QOL) and recovery, as reflected by less postoperative pain, shorter hospital stay, quicker return to normal activities, higher QOL values and better cosmetic results when compared to open surgery. ${ }^{1-10}$ The United Nations and the WHO have both written reports on the rapid population aging, stating that, by 2050 , one in five people will be at least 60 years old. ${ }^{11,12}$ With this growing elderly population, more patients will undergo surgery at an older age. Data are becoming available on elderly patients' recovery and outcome after minimally invasive procedures. A recent overview of oncological procedures shows that with advanced age, major surgery is a risk factor for cognitive decline, ${ }^{13}$ in this light, a laparoscopic approach is potentially favorable in the elderly.

In gynecology, a woman's lifetime risk of undergoing a hysterectomy is around $10 \%-30 \% .{ }^{14}$ Ninety percent of all hysterectomies are performed for non-malignant 
conditions to improve the patient's health-related QOL. ${ }^{14}$ A hysterectomy has shown to be successful in improving QOL in women suffering from gynecological disorders. ${ }^{15-18}$ The percentage of hysterectomies that are performed laparoscopically in the developed world has increased lately, ${ }^{19,20}$ while the total number of hysterectomies performed to treat benign conditions is decreasing due to less-invasive alternatives. ${ }^{21}$ When comparing laparoscopic hysterectomy (LH) with abdominal hysterectomy $(\mathrm{AH})$ for benign indications, studies report a better and faster short-term improvement in QOL after the laparoscopic approach. ${ }^{3,4}$

A growing proportion of older women will have a malignant indication for hysterectomy, as with increasing age and body mass index (BMI) the incidence of endometrial cancer will increase. ${ }^{22}$ In early-stage low-risk endometrial cancer, several reviews report a better short-term QOL and faster recovery after $\mathrm{LH}$ compared to $\mathrm{AH} .^{5-10}$ Moreover, $\mathrm{LH}$ is regarded as being just as safe as $\mathrm{AH} .{ }^{5,8-10} \mathrm{~A}$ recent retrospective review reported that, specifically in endometrial cancer patients $>60$ years of age, the laparoscopic approach was associated with decreased morbidity even when surgical staging was performed, compared with laparotomy. ${ }^{23}$ It was also shown that with increasing age the benefit of a laparoscopic approach was even higher due to more complications in the laparotomy group $\geq 60$ years of age. ${ }^{23}$ Another report on a large cohort of endometrial cancer patients showed that the elderly (defined as $\geq 65$ or $\geq 75$ years of age) had more complications in the laparotomy group compared with the laparoscopy group. ${ }^{24}$ In these reports, the benefits of the laparoscopic approach with increasing age have been described in terms of surgical outcome, but they do not describe the impact on QOL in this specific population.

The aim of this prospective cohort study was to determine the impact of LH on QOL and recovery over 6 months time, specifically in older patients. All QOL values of patients were compared pre- vs postoperatively and to the mean in the group; in addition, they were compared with an unselected, age-matched female reference population.

\section{Patients and methods}

\section{Study design and patients}

A multicenter, prospective, single-arm cohort study that served as a preparation for a large randomized controlled trial was conducted in the north of the Netherlands at the University Medical Center Groningen (UMCG) and seven regional hospitals (three teaching and four non-teaching hospitals) from January 2005 until January 2007. ${ }^{25}$ Eleven general gynecologists participated. All patients were operated on according to a standardized operation protocol, as described in a previous report. ${ }^{26}$ The Medical Ethical Committee of the UMCG approved the study protocol.

Consecutive patients with a benign indication for hysterectomy or with early-stage low-risk endometrial cancer, 18 years of age and older, were included. Benign indications for hysterectomy included dysfunctional uterine bleeding, menstrual disorders, and myomas. Early-stage low-risk endometrial cancer was defined as histologically proven endometrioid adenocarcinoma, grade 1 or 2 , or complex atypical hyperplasia, clinically confined to the uterine corpus, without cervical involvement (ie, clinical stage I). Exclusion criteria included histological types other than grade 1 or 2 endometrioid adenocarcinoma, clinically advanced disease, uterus size larger than expected at 12-week pregnancy, and cardiopulmonary contraindications for laparoscopy. Figure 1 shows the flowchart for inclusions and exclusions in this study. All patients signed a written informed consent form before participating.

\section{Variables measured}

QOL was measured using the 36-item Short Form Health Survey (SF-36) questionnaire preoperatively. QOL was then measured again 6 weeks postoperatively and then 6 months postoperatively, following the European guidelines, which state that in case of a hysterectomy QOL should be assessed using the SF-36 within at least 6 months. ${ }^{27}$ The SF-36 consists of 36 questions with standardized response choices that are organized in 8 scales: physical functioning, role limitations due to physical health problems, bodily pain, general health, vitality, social functioning, role limitations due to emotional problems, and mental health. All scales are linearly converted to a 0-100 scale. Higher scores indicate higher levels of functioning or well-being. The SF-36 has been translated into Dutch, and this version has been validated. ${ }^{28}$

On a case record form (CRF), the following patient characteristics were recorded: age, BMI, complications during the operation or discovered within 6 weeks postoperatively, the amount of blood loss in milliliters, conversions from laparoscopy to laparotomy and reason for conversion, use of pain medication postoperatively, and length of hospital stay after surgery. Complications were classified as major or minor complications according to the Common Terminology Criteria of Adverse Events version 3.0 (http://ctep.cancer. gov/protocolDevelopment/electronic applications/docs/ ctcaev3.pdf). The severity of a complication was judged by an independent panel of medical doctors, described in detail previously. ${ }^{26}$ 


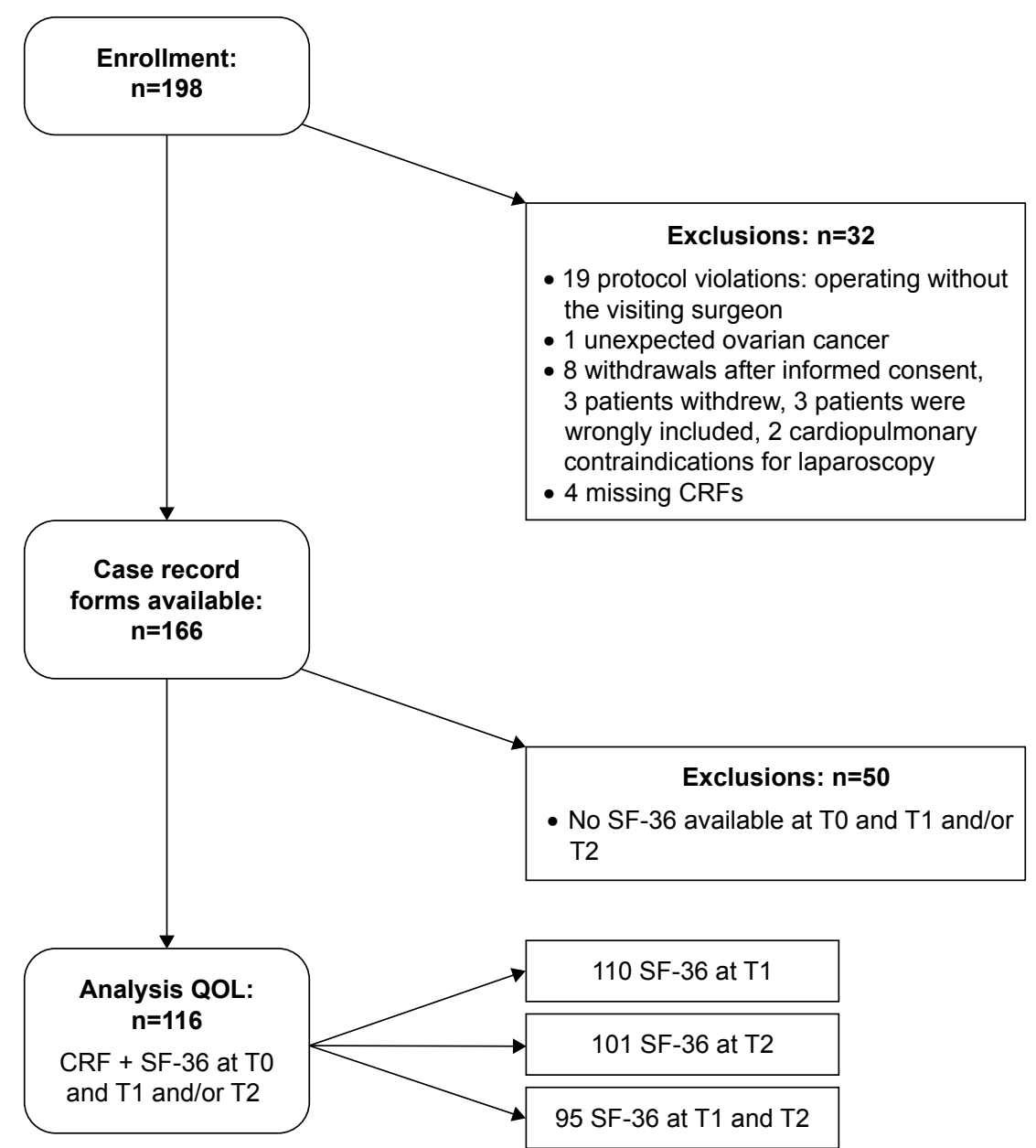

Figure I Flowchart for this prospective multicenter cohort study.

Abbreviations: CRF, case record form; SF-36, 36-item Short Form Health Survey; T0, before the operation; TI, 6 weeks after the operation; T2, 6 months after the operation; QOL, quality of life.

\section{Reference population}

As a reference, an unselected population of Dutch women in the age group of the study population was available for comparison of the SF-36 data. ${ }^{28}$ These data were collected from a study that was undertaken to validate the SF-36 in the Dutch language and to create normative data of the SF-36 among Dutch-speaking residents of the Netherlands.

\section{Statistical analysis}

Patients were included in the analysis if at least the preoperative SF-36 and one of the postoperative SF-36 data were available. Patient characteristics as well as clinical outcome were compared for the patients who did and did not return the SF-36s. In addition, younger patients ( $<65$ years of age) were compared with older patients ( $\geq 65$ years of age). Differences between groups were tested using the chi-squared test or the Mann-Whitney test, and significance was set at $P<0.05$ (two-tailed). Descriptive statistics (mean and SDs) for the QOL data were calculated and plotted for each assessment for the patients included in this analysis. Younger patients ( $<65$ years of age) were compared with older patients ( $\geq 65$ years of age) regarding their score on the SF-36. To assess the changes in QOL scores at 6 weeks and 6 months after baseline and to identify subgroups of patients who have a QOL below what could be expected, a longitudinal linear mixed model was applied. An unstructured covariance matrix was assumed. Covariates in the longitudinal model were preselected by using univariate linear regression. In this regression analysis, change in QOL score, at the 6-week follow-up and at the 6-month follow-up, was the dependent variable and the covariates from Table 1 were the independent variables. A covariate was considered statistically significant when the associated $P$-value was below 0.25 at either the 6-week follow-up or the 6-month follow-up. Backward elimination of the selected covariates and their interaction terms with follow-up time (restricting to hierarchical models only) was applied to form the final model. In this analysis, $P<0.01$ was considered statistically significant. All tests were 
Table I Patient characteristics and clinical outcome of II6 patients included in this study compared to the 50 patients who did not return the SF-36s

\begin{tabular}{|c|c|c|c|}
\hline & $\begin{array}{l}\text { Included in study } \\
(\mathrm{N}=\mid 16)\end{array}$ & $\begin{array}{l}\text { Not included in study } \\
(\mathrm{N}=50)\end{array}$ & $P$-value \\
\hline Age (years), median (IQR) & $54(43-65)$ & $51(44-67)$ & 0.64 \\
\hline $\begin{array}{l}\text { Body mass index }\left(\mathrm{kg} / \mathrm{m}^{2}\right) \\
\quad<25 \\
25-29.9 \\
>30\end{array}$ & $\begin{array}{l}31(28.4) \\
42(38.5) \\
36(33.0)\end{array}$ & $\begin{array}{l}12(25.5) \\
16(34) \\
16(34)\end{array}$ & 0.95 \\
\hline Previous laparotomy & $27(23.7)$ & $8(17.8)$ & 0.42 \\
\hline Comorbidity & $40(38.1)$ & $18(38.3)$ & 0.98 \\
\hline Malignant indication & $52(44.8)$ & $25(50)$ & 0.54 \\
\hline Hospital stay (days), median (IQR) & $2(2-3)$ & $2(2-4)$ & 0.46 \\
\hline Major complications & $4(3.4)$ & $4(8.0)$ & 0.24 \\
\hline Minor complications & $18(15.5)$ & $2(4.0)$ & 0.04 \\
\hline Conversions to laparotomy & II (9.5) & $9(18)$ & 0.12 \\
\hline Operating time, median (IQR) & $\mathrm{I}: 55$ hours (1:40-2:30 hours) & $2: 10$ hours ( $1: 30-2: 35$ hours) & 0.19 \\
\hline Blood loss, median (IQR) & $150 \mathrm{~mL}(80-200 \mathrm{~mL})$ & $200 \mathrm{~mL}(\mathrm{I} 00-300 \mathrm{~mL})$ & 0.24 \\
\hline No pain medication needed after postoperative day 2 & $74(63.8)$ & $26(56.5)$ & 0.39 \\
\hline
\end{tabular}

Notes: Data shown as $\mathrm{n}(\%)$ unless otherwise specified. A value in bold shows a significant difference between the two groups.

Abbreviations: IQR, interquartile range; SF-36, 36-item Short Form Health Survey.

two-sided. Analyses were performed using the SPSS 16.02 software for Windows XP and SAS software.

\section{Results}

\section{Baseline characteristics}

A total of 116 out of 166 patients $(70 \%)$ returned the preoperative SF-36 and at least one of the postoperative SF-36 questionnaires. Of these 116 patients, 110 (95\%) patients returned the 6-week SF-36 and 101 (87\%) the 6-month
SF-36. A total of 95 patients $(82 \%)$ returned the 6-week as well as the 6-month SF-36 (Figure 1). The median age of the women included in this study was 54 years (range 34-83; interquartile range [IQR]: 43-65). Of the 116 patients, 83 were $<65$ years of age and 26 were $\geq 65$ years of age at time of surgery (missing data $n=7$ ). In both groups, $70 \%$ or more patients were overweight or obese with a median BMI of $27 \mathrm{~kg} / \mathrm{m}^{2}$ (Table 2). A total of 64 (55\%) patients had earlystage low-risk endometrial cancer, and 52 (45\%) patients

Table 2 Patient characteristics and clinical outcome of I 16 patients included in this study stratified by age

\begin{tabular}{|c|c|c|c|}
\hline & $\begin{array}{l}\text { Younger patients } \\
\text { ( }<65 \text { years), } \mathbf{N}=83\end{array}$ & $\begin{array}{l}\text { Older patients } \\
\text { ( } \geq 65 \text { years), } N=26\end{array}$ & $P$-value \\
\hline Age (years), median (IQR) & $48(43-57)$ & $72(68-77)$ & $<0.001$ \\
\hline $\begin{array}{l}\text { Body mass index }\left(\mathrm{kg} / \mathrm{m}^{2}\right) \\
\quad<25 \\
25-29.9 \\
>30\end{array}$ & $\begin{array}{l}25(30.1) \\
31(37.3) \\
27(32.5)\end{array}$ & $\begin{array}{l}6(23.1) \\
11(42.3) \\
9(34.6)\end{array}$ & 0.50 \\
\hline Previous laparotomy & $18(20.5)$ & $9(34.6)$ & 0.14 \\
\hline Comorbidity & $28(34.1)$ & $12(52.2)$ & 0.12 \\
\hline Malignant indication & $29(32.2)$ & $23(88.5)$ & $<0.001$ \\
\hline Hospital stay (days), median (IQR) & $2(2-3)$ & $2.5(2-4)$ & 0.49 \\
\hline Major complications & $4(4.4)$ & 0 & 0.27 \\
\hline Minor complications & II (12.2) & $7(26.9)$ & 0.07 \\
\hline Conversions to laparotomy & II (12.2) & 0 & 0.06 \\
\hline Operating time, median (IQR) & $1: 50$ hours ( $1: 40-2: 15$ hours) & $2: 30$ hours ( $1: 47-2: 43$ hours) & 0.009 \\
\hline Blood loss, median (IQR) & $125 \mathrm{~mL}(50-212 \mathrm{~mL})$ & $150 \mathrm{~mL}(100-200 \mathrm{~mL})$ & 0.86 \\
\hline No pain medication needed after postoperative day 2 & $59(65.6)$ & I5 (57.7) & 0.46 \\
\hline
\end{tabular}

Notes: Data shown as $\mathrm{n}(\%)$ unless otherwise specified. A value in bold shows a significant difference between the younger and older group.

Abbreviation: IQR, interquartile range. 
had a benign indication for hysterectomy. There were no statistically significant differences observed between the characteristics of the patients included in the study $(n=116)$ and the 50 patients who did not return the SF-36 (Table 1).

\section{Outcome}

In the study population, the median hospital stay was 2 days (IQR 2-3 days) and the median operating time was 1:55 hours (IQR 1:40-2:30 hours). Four major complications occurred in the 116 patients included in this study (3.4\%) and four major complications occurred in the 50 patients who did not return the SF-36 (8.0\%). There were no statistically significant differences observed between the responding and non-responding groups, except for the rate of minor complications, which was higher in the responding group (15.5\% vs $4.0 \%$, Table 1 ).

When comparing the patients $<65$ years of age and $\geq 65$ years of age at time of surgery, conversions to laparotomy occurred more often in the younger group, though not statistically significant ( 11 vs $0 P=0.06$ ). In the older group, more patients had early-stage endometrial cancer (23 [88\%] vs 29 [32\%], $P<0.001)$. Operating time was significantly longer in older patients (median 2.30 vs 1.50 hours $P=0.009$ ) (Table 2).

\section{Outcome measure - QOL}

All mean subscale values in the study population were significantly higher than the baseline values (Figure 2) 6 months after the operation. Compared with the reference population before the operation, patients scored significantly lower on general health, social functioning, and role limitations due to emotional problems. After 6 weeks, the patients scored significantly lower on role limitations due to physical problems, but significantly higher on mental health and bodily pain (indicating less pain). All mean scores were above the reference population values 6 months after the operation. On the subscale general health, scores were equal to the reference values. On the subscales social functioning, role limitations due to physical problems, mental health, vitality, and bodily pain, this was a significant positive difference.

Older patients scored relatively high before the operation and showed significantly less change in QOL over time, when comparing preoperative and postoperative QOL values on physical functioning, social functioning, role limitations due to physical problems, vitality, bodily pain, and general health (Table 3). After a major complication, patients $(n=4)$ scored significantly higher on physical functioning and bodily pain at 6 months compared with those without a major complica- tion; indicating better physical functioning and less bodily pain (Table 4). Patients suffering a minor complication $(n=18)$ scored significantly higher on bodily pain at 6 months, indicating less pain than those without a minor complication. An overview of the regression coefficients of the model is provided in Table $\mathrm{S} 1$.

\section{Discussion}

In patients with benign conditions or early-stage low-risk endometrial cancer, LH has a positive impact on QOL as measured prospectively by the SF-36 up to 6 months postoperatively. All features of QOL are significantly improved at 6 months compared with preoperative values and are better than or equal to the reference population values. In older patients ( $\geq 65$ years of age), QOL improves as well; these patients reported relatively high preoperative QOL values and their QOL values remain high over time postoperatively up to 6 months.

Our findings of improved QOL after LH are in accordance with the findings of others. ${ }^{3,4}$ Previous studies have also found that LH results in better short-term QOL and faster recovery compared with $\mathrm{AH}$ in benign conditions ${ }^{3,4}$ as well as in early-stage low-risk endometrial cancer. ${ }^{5-10}$ To the best of our knowledge, our observations that older patients exhibit higher SF-36 QOL scores and little change in QOL up to 6 months following an LH, evaluated prospectively, have not been described before. In open gynecologic surgery, elderly patients have been found to show little change in QOL over time from preoperatively to postoperatively. ${ }^{29}$ According to the U-bend theory, older people tend to be happier than younger people. ${ }^{30}$ According to this theory, older people are better at controlling emotions and accepting misfortune; this may be why the elderly reported less change in QOL after surgery.

Following surgery, minimal side effects are especially important for elderly patients, as comorbidity such as diabetes or cardiovascular disease is often present. A laparotomy is often "major" surgery, with a larger wound, a higher risk of wound complications, more pain, and a longer hospital stay, which are all described as risk factors for cognitive decline in the elderly. ${ }^{13}$ The inflammatory response (toxicity) is higher following laparotomy, resulting in a slower recovery, and this possibly lowers survival due to the patient's inability to undergo additional therapy. ${ }^{23}$ Again, laparoscopic surgery is the less invasive and hence the preferred route.

As the number of people at a higher age is growing, there will be an increase in the number of elderly people needing surgery. Moreover, an increase in the number of cases of 

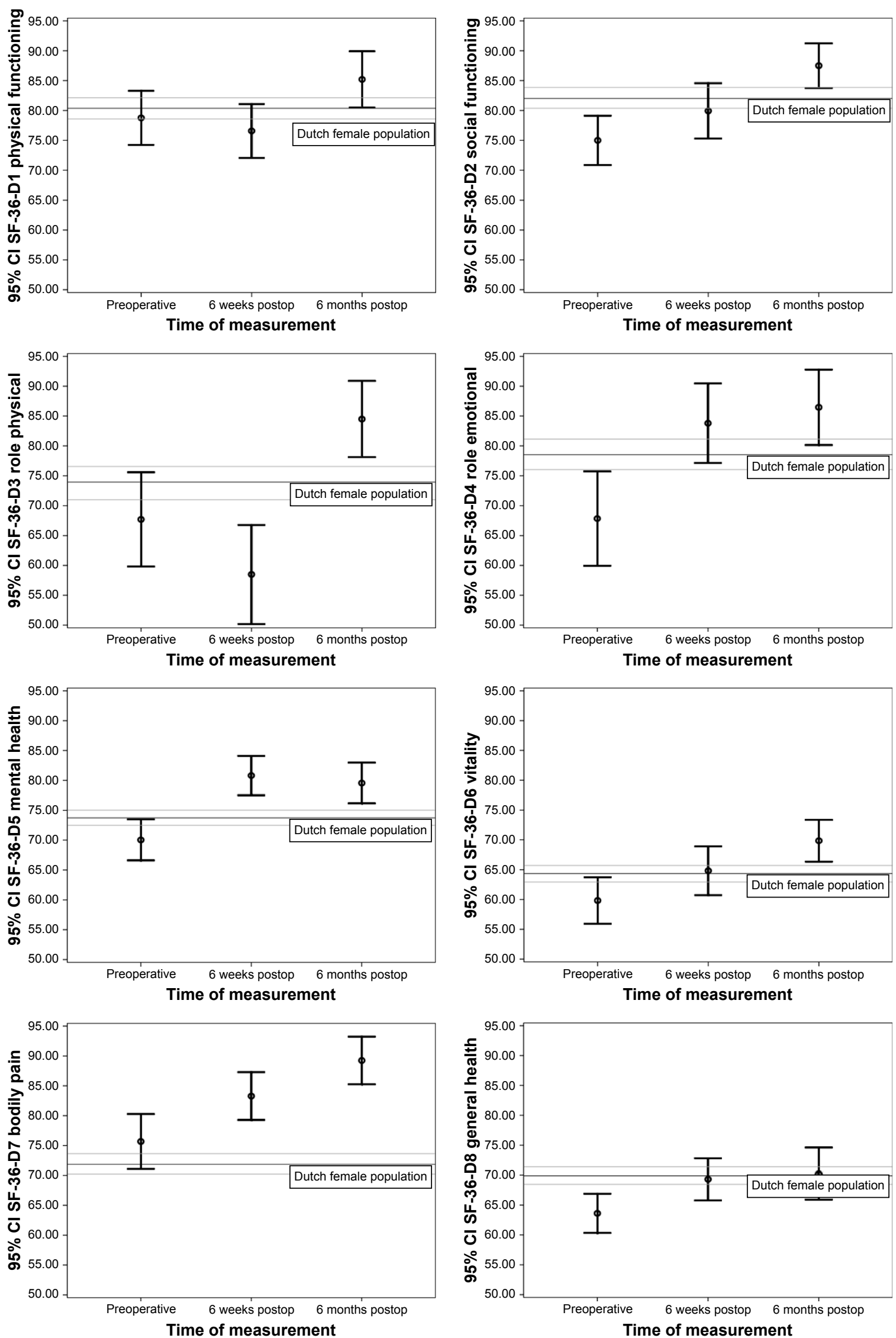

Figure 2 The eight dimensions on the SF-36 in 116 patients over time compared to the reference population.

Note: Between the light gray lines is the $\mathrm{Cl}$ of the values for the Dutch female (reference) population.

Abbreviations: D, SF-36 domain number; postop, postoperative; SF-36, 36-item Short Form Health Survey. 
Table 3 Scores on the SF-36, Dimensions I-8 stratified by age (average with $95 \% \mathrm{Cls}$ )

\begin{tabular}{|c|c|c|c|c|c|c|}
\hline \multirow[t]{2}{*}{ SF-36 domain } & \multicolumn{3}{|c|}{ Younger patients ( $<65$ years) } & \multicolumn{3}{|c|}{ Older patients ( $\geq 65$ years) } \\
\hline & Preoperatively & 6 weeks $^{a}$ & 6 months $^{b}$ & Preoperatively & 6 weeks $^{a}$ & 6 months ${ }^{\mathrm{b}}$ \\
\hline Physical functioning & $79.9(75.0 ; 84.9)$ & $-0.4(-7.5 ; 6.8)$ & $8.9(1.6 ; 16.2)$ & $75.2(66.5 ; 83.9)$ & $-8.1(-20.6 ; 4.5)$ & $-1.9(-14.9 ; 11.0)$ \\
\hline Social functioning & $71.4(66.8 ; 76.0)$ & $8.3(1.7 ; 14.9)$ & I5.6 (8.8; 22.4) & $85.8(77.8 ; 93.8)$ & $-5.2(-16.7 ; 6.3)$ & $3.3(-8.6 ; 15.2)$ \\
\hline Role physical & $65.6(57.1 ; 74.1)$ & $-8.3(-20.4 ; 3.8)$ & $19.9(7.6 ; 32.3)$ & $74.1(59.3 ; 88.9)$ & $-11.6(-33.4 ; 10.2)$ & $7.1(-14.6 ; 28.9)$ \\
\hline Role emotional & $67.4(59.6 ; 75.3)$ & I7.I (5.9; 28.4) & $20.7(9.2 ; 32.2)$ & $69.0(55.3 ; 82.8)$ & $12.3(-7.8 ; 32.3)$ & $12.3(-7.8 ; 32.3)$ \\
\hline Mental health & $69.6(65.8 ; 73.4)$ & $11.3(5.9 ; 16.7)$ & $10.2(4.7 ; 15.8)$ & $71.3(64.8 ; 77.8)$ & $9.2(-0.3 ; 18.6)$ & $7.4(-2.2 ; 17.0)$ \\
\hline Vitality & $58.1(53.8 ; 62.4)$ & $5.9(-0.3 ; 12.0)$ & $12.0(5.8 ; 18.3)$ & $65.0(57.6 ; 72.4)$ & $2.5(-8.3 ; 13.3)$ & $4.0(-6.9 ; 14.9)$ \\
\hline Bodily pain & $72.9(68.2 ; 77.6)$ & $9.5(2.7 ; 16.2)$ & I5.8 $(9.0 ; 22.7)$ & $83.9(75.8 ; 92.0)$ & $2.0(-9.5 ; 13.6)$ & $6.9(-5.1 ; 18.9)$ \\
\hline General health & $64.7(60.7 ; 68.8)$ & $5.2(-0.7 ; 11.0)$ & $7.4(1.4 ; 13.3)$ & $60.2(53.1 ; 67.3)$ & $7.4(-2.8 ; 17.6)$ & $3.9(-6.8 ; 14.7)$ \\
\hline
\end{tabular}

Notes: ${ }^{a} 6$ weeks postoperatively compared with preoperatively; ${ }^{b} 6$ months postoperatively compared with preoperatively.

Abbreviations: D, SF-36 domain number; SF-36, 36-item Short Form Health Survey.

endometrial cancer is expected due to aging and increase in BMI in the population. ${ }^{22}$ Therefore, the beneficial effect of LH on QOL and the minimal impact of age on QOL after LH should be taken into account when considering surgical treatment for elderly patients.

In our design, we included both benign and malignant indications for a hysterectomy. This allowed us to prospectively include all patients undergoing an $\mathrm{LH}$, following the same procedure according to a strict surgical protocol. The strength of our study is that we simultaneously and prospectively collected QOL data during the implementation of a minimally invasive gynecological procedure. In addition, we compared our values with a set of values from a reference population. We made this comparison to gain insight into the QOL scores of our study population. However, we should take into account that our patients completed these questionnaires around a surgical intervention, as opposed to the reference population who did not undergo an intervention.

These data were collected in an implementation setting, and the number of patients available for analysis was limited. Regarding age, a continuous variable was used because splitting patients into different age groups resulted in groups that were too small to compare. Although our data are over 10 years old, the information is still interesting as it shows that, with respect to QOL, the laparoscopic route is favorable even in the elderly. Moreover, as LH is nowadays a common practice and routine, one could expect even higher QOL values as fewer complications occur when surgical teams are more experienced and are using smarter surgical devices and techniques.

\section{Conclusion}

In this multicenter prospective study in which all patients were operated on according to the same operation protocol, all QOL values improved significantly in 6 months following LH. Older patients' QOL values hardly change from the preoperative period to 6 months after the operation, which demonstrates that the laparoscopic procedure has no negative impact on QOL. Therefore, all women with an indication for an $\mathrm{AH}$ should be considered for a laparoscopic procedure, regardless of age.

\section{Ethical approval}

The study was approved by the Medical Ethical Committee of the University Medical Center Groningen and conducted according to the principles of the Declaration of Helsinki and in accordance with the Medical Research Involving Human Subjects Act (Wet medisch-wetenschappelijk onderzoek met mensen [WMO]).

Table 4 Significantly different QOL in subgroups of patients compared to the mean in the group

\begin{tabular}{|c|c|c|c|}
\hline \multirow[t]{2}{*}{ Subgroup of patients } & \multicolumn{3}{|l|}{ Scales on the SF-36 } \\
\hline & Preoperatively & 6 weeks postoperatively & 6 months postoperatively \\
\hline $\begin{array}{l}\text { Indication early stage endometrial } \\
\text { cancer }(\mathrm{n}=52)\end{array}$ & $\begin{array}{l}\text { (:) Physical functioning } \\
\text { :) Vitality }\end{array}$ & & \\
\hline Major complication $(n=4)$ & & $\begin{array}{l}\text { : Physical functioning } \\
\text { : Bodily pain }\end{array}$ & $\begin{array}{l}\text { (:) Physical functioning } \\
\text { (:) Bodily pain }\end{array}$ \\
\hline Minor complication $(n=18)$ & & (2) Bodily pain & (): Bodily pain \\
\hline Present comorbidity $(n=40)$ & ;:) Bodily pain & : : General health & \\
\hline
\end{tabular}

Notes: Smiley face represents better scores; frowning face represents worse scores.

Abbreviations: QOL, quality of life; SF-36, 36-item Short Form Health Survey. 


\section{Acknowledgments}

We would like to thank the following participating gynecologists and centers for patient recruitment: Antonius Ziekenhuis Sneek, Isala Klinieken Zwolle, Leveste Ziekenhuis Emmen, Martini Ziekenhuis Groningen, Medisch Centrum Leeuwarden, Nij Smellinghe Drachten, Universitair Medisch Centrum Groningen, and Wilhelmina Ziekenhuis Assen.

An abstract of this paper was presented as an e-poster presentation with interim findings at the 19th International Meeting of the European Society of Gynecological Oncology; October 24-27, 2015; Nice, France.

This study was supported by a grant from the University Medical Center Groningen, Groningen, the Netherlands.

\section{Author contributions}

JMB, GHdB, and MJEM contributed to conception and design. JMB and MJAK acquired the data. JMB, GHdB, ERvdH, and MJEM contributed to the analysis and interpretation of the data. All authors contributed toward data analysis, drafting and critically revising the paper, gave final approval of the version to be published, and agree to be accountable for all aspects of the work.

\section{Disclosure}

The authors report no conflicts of interest in this work.

\section{References}

1. Wei B, Qi CL, Chen TF, et al. Laparoscopic versus open appendectomy for acute appendicitis: a metaanalysis. Surg Endosc. 2011;25(4): 1199-1208.

2. McGinn FP, Miles AJ, Uglow M, Ozmen M, Terzi C, Humby M. Randomized trial of laparoscopic cholecystectomy and mini-cholecystectomy. Br J Surg. 1995;82(10):1374-1377.

3. Nieboer TE, Hendriks JC, Bongers MY, Vierhout ME, Kluivers KB. Quality of life after laparoscopic and abdominal hysterectomy: a randomized controlled trial. Obstet Gynecol. 2012;119(1):85-91.

4. Kluivers KB, Hendriks JC, Mol BW, et al. Quality of life and surgical outcome after total laparoscopic hysterectomy versus total abdominal hysterectomy for benign disease: a randomized, controlled trial. J Minim Invasive Gynecol. 2007;14(2):145-152.

5. Kluivers KB, Ten Cate FA, Bongers MY, Brölmann HA, Hendriks JC. Total laparoscopic hysterectomy versus total abdominal hysterectomy with bilateral salpingo-oophorectomy for endometrial carcinoma: a randomised controlled trial with 5-year follow-up. Gynecol Surg. 2011;8(4): 427-434.

6. Kornblith AB, Huang HQ, Walker JL, Spirtos NM, Rotmensch J, Cella D. Quality of life of patients with endometrial cancer undergoing laparoscopic international federation of gynecology and obstetrics staging compared with laparotomy: a Gynecologic Oncology Group study. J Clin Oncol. 2009;27(32):5337-5342.

7. Janda M, Gebski V, Brand A, et al. Quality of life after total laparoscopic hysterectomy versus total abdominal hysterectomy for stage I endometrial cancer (LACE): a randomised trial. Lancet Oncol. 2010;11(8):772-780.

8. Janda M, Gebski V, Davies LC, et al. Effect of total laparoscopic hysterectomy vs total abdominal hysterectomy on disease-free survival among women with stage 1 endometrial cancer: a randomized clinical trial. JAMA. 2017;317(12):1224-1233.
9. Mourits MJ, Bijen CB, Arts HJ, et al. Safety of laparoscopy versus laparotomy in early-stage endometrial cancer: a randomised trial. Lancet Oncol. 2010;11(8):763-771.

10. Zullo F, Falbo A, Palomba S. Safety of laparoscopy vs laparotomy in the surgical staging of endometrial cancer: a systematic review and metaanalysis of randomized controlled trials. Am J Obstet Gynecol. 2012;207(2):94-100

11. United Nations. World Population Ageing 1950-2050 [2002]. Available from: http://www.un.org/esa/population/publications/worldageing 19502050/. Accessed November 11, 2018.

12. World Health Organization. Ageing and health [February 5, 2018]. Available from: http://www.who.int/mediacentre/factsheets/fs404/en/. Accessed November 11, 2018.

13. Plas M, Rotteveel E, Izaks GJ, et al. Cognitive decline after major oncological surgery in the elderly. Eur J Cancer. 2017;86:394-402.

14. Rock JA. Quality-of-life assessment in gynecologic surgery. J Reprod Med. 2001;46(5 Suppl):515-519.

15. Rannestad T, Eikeland OJ, Helland H, Qvarnström U. The quality of life in women suffering from gynecological disorders is improved by means of hysterectomy. Absolute and relative differences between pre- and postoperative measures. Acta Obstet Gynecol Scand. 2001; 80(1):46-51.

16. Thakar R, Ayers S, Georgakapolou A, Clarkson P, Stanton S, Manyonda I. Hysterectomy improves quality of life and decreases psychiatric symptoms: a prospective and randomised comparison of total versus subtotal hysterectomy. BJOG. 2004;111(10):1115-1120.

17. Summitt RL Jr, Kuppermann M, Varner RE; Ms Research Group. Effect of hysterectomy vs medical treatment on health-related quality of life and sexual functioning: the medicine or surgery (Ms) randomized trial. JAMA. 2004;291(12):1447-1455.

18. Radosa JC, Meyberg-Solomayer G, Kastl C, et al. Influences of different hysterectomy techniques on patients' postoperative sexual function and quality of life. $J$ Sex Med. 2014;11(9):2342-2350.

19. Twijnstra AR, Kolkman W, Trimbos-Kemper GC, Jansen FW. Implementation of advanced laparoscopic surgery in gynecology: national overview of trends. J Minim Invasive Gynecol. 2010;17(4): 487-492.

20. Turner LC, Shepherd JP, Wang L, Bunker CH, Lowder JL. Hysterectomy surgery trends: a more accurate depiction of the last decade? Am J Obstet Gynecol. 2013;208(4):277.e1-277.e7.

21. Fergusson RJ, Lethaby A, Shepperd S, Farquhar C. Endometrial resection and ablation versus hysterectomy for heavy menstrual bleeding. Cochrane Database Syst Rev. 2013;11:CD000329.

22. Hagemann AR, Mccourt CK, Varaday SS, Moore KN. Defining and mitigating the challenges of an older and obese population in minimally invasive gynecologic cancer surgery. Gynecol Oncol. 2018;148(3): 601-608.

23. Bishop EA, Java JJ, Moore KN, et al. Surgical outcomes among elderly women with endometrial cancer treated by laparoscopic hysterectomy: a NRG/Gynecologic Oncology Group study. Am J Obstet Gynecol. 2018; 218(1):109.e1-109.e11.

24. Uccella S, Bonzini M, Palomba S, et al. Laparoscopic vs. open treatment of endometrial cancer in the elderly and very elderly: an age-stratified multicenter study on 1606 women. Gynecol Oncol. 2016;141(2): 211-217.

25. Bijen CB, BriëtJM, deBock GH, Arts HJ, Bergsma-KadijkJA, Mourits MJ. Total laparoscopic hysterectomy versus abdominal hysterectomy in the treatment of patients with early stage endometrial cancer: a randomized multi center study. BMC Cancer. 2009;9:23.

26. Briët JM, Mourits MJ, Kenkhuis MJ, van der Zee AG, de Bock GH, Arts HJ. Implementing an advanced laparoscopic procedure by monitoring with a visiting surgeon. J Minim Invasive Gynecol. 2010;17(6): 771-778.

27. Korolija D, Sauerland S, Wood-Dauphinée S, et al; European Association for Endoscopic Surgery. Evaluation of quality of life after laparoscopic surgery: evidence-based guidelines of the European Association for Endoscopic Surgery. Surg Endosc. 2004;18(6):879-897. 
28. Aaronson NK, Muller M, Cohen PD, et al. Translation, validation, and norming of the Dutch language version of the SF-36 Health Survey in community and chronic disease populations. J Clin Epidemiol. 1998;51(11):1055-1068.

29. von Gruenigen VE, Gil KM, Frasure HE, Jenison EL, Hopkins MP. The impact of obesity and age on quality of life in gynecologic surgery. Am J Obstet Gynecol. 2005;193(4):1369-1375.
30. The Economist. Age and happiness; The U-bend of life [December 16, 2010]. Available from: http://www.economist.com/node/17722567. Accessed November 11, 2018 


\section{Supplementary material}

Table SI Significantly different QOL in subgroups of patients compared to the mean in the group: regression coefficients

\begin{tabular}{|c|c|c|c|c|c|c|}
\hline SF-36 domain & Period & Age & $\begin{array}{l}\text { Indication early } \\
\text { stage EC }\end{array}$ & $\begin{array}{l}\text { Major } \\
\text { complication }\end{array}$ & $\begin{array}{l}\text { Minor } \\
\text { complication }\end{array}$ & $\begin{array}{l}\text { Present } \\
\text { comorbidity }\end{array}$ \\
\hline \multirow[t]{3}{*}{ Physical functioning } & Pre $^{b}$ & -0.47 & 15.96 & & & \\
\hline & 6 weeks $^{c}$ & -0.34 & & -29.31 & & \\
\hline & 6 months $^{d}$ & -0.49 & & -39.21 & & \\
\hline \multirow[t]{3}{*}{ Social functioning } & $\mathrm{Pre}^{\mathrm{b}}$ & 0.75 & & & & \\
\hline & 6 weeks $^{c}$ & -0.43 & & & & \\
\hline & 6 months $^{d}$ & -0.63 & & & & \\
\hline \multirow[t]{3}{*}{ Role physical } & Pre $^{a}$ & 0.49 & & & & \\
\hline & 6 weeks $^{c}$ & & & & & \\
\hline & 6 months $^{d}$ & -0.63 & & & & \\
\hline \multirow[t]{3}{*}{ Vitality } & Pre $^{b}$ & 0.25 & 8.61 & & & \\
\hline & 6 weeks $^{c}$ & & & & & \\
\hline & 6 months $^{d}$ & -0.46 & & & & \\
\hline \multirow[t]{3}{*}{ Bodily pain } & Pre $^{b}$ & 0.67 & & & & 7.67 \\
\hline & 6 weeks $^{c}$ & -0.47 & & -31.73 & -19.52 & \\
\hline & 6 months $^{d}$ & -0.67 & & 44.35 & 13.26 & \\
\hline \multirow[t]{3}{*}{ General health } & Pre $^{b}$ & 0.16 & & & & -3.34 \\
\hline & 6 weeks $^{c}$ & & & & & -7.24 \\
\hline & 6 months $^{d}$ & -0.39 & & & & \\
\hline
\end{tabular}

Notes: aEarly stage endometrial cancer (EC); ${ }^{b}$ preoperatively; ${ }^{\circ} 6$ weeks postoperatively compared to preoperatively; ${ }^{\mathrm{d}} 6$ months postoperatively compared to preoperatively. Abbreviations: QOL, quality of life; SF-36, 36-item Short Form Health Survey.

Clinical Interventions in Aging

\section{Publish your work in this journal}

Clinical Interventions in Aging is an international, peer-reviewed journal focusing on evidence-based reports on the value or lack thereof of treatments intended to prevent or delay the onset of maladaptive correlates of aging in human beings. This journal is indexed on PubMed Central, MedLine,

\section{Dovepress}

CAS, Scopus and the Elsevier Bibliographic databases. The manuscript management system is completely online and includes a very quick and fair peer-review system, which is all easy to use. Visit http://www.dovepress. $\mathrm{com} /$ testimonials.php to read real quotes from published authors. 